\title{
Using Tractography to Distinguish SWEDD from Parkinson's Disease Patients Based on Connectivity
}

\author{
Mansu Kim ${ }^{1}$ and Hyunjin Park ${ }^{2,3}$ \\ ${ }^{1}$ Department of Electronic, Electrical and Computer Engineering, Sungkyunkwan University, Suwon 16419, Republic of Korea \\ ${ }^{2}$ School of Electronic and Electrical Engineering, Sungkyunkwan University, Suwon 16419, Republic of Korea \\ ${ }^{3}$ Center for Neuroscience Imaging Research (CNIR), Institute for Basic Science, Suwon 16419, Republic of Korea \\ Correspondence should be addressed to Hyunjin Park; hyunjinp@skku.edu
}

Received 28 October 2015; Revised 3 February 2016; Accepted 10 February 2016

Academic Editor: Carlo Colosimo

Copyright (C) 2016 M. Kim and H. Park. This is an open access article distributed under the Creative Commons Attribution License, which permits unrestricted use, distribution, and reproduction in any medium, provided the original work is properly cited.

\begin{abstract}
Background. It is critical to distinguish between Parkinson's disease (PD) and scans without evidence of dopaminergic deficit (SWEDD), because the two groups are different and require different therapeutic approaches. Objective. The aim of this study was to distinguish SWEDD patients from PD patients using connectivity information derived from diffusion tensor imaging tractography. Methods. Diffusion magnetic resonance images of SWEDD $(n=37)$ and PD $(n=40)$ were obtained from a research database. Tractography, the process of obtaining neural fiber information, was performed using custom software. Group-wise differences between PD and SWEDD patients were quantified using the number of connected fibers between two regions, and correlation analyses were performed based on clinical scores. A support vector machine classifier (SVM) was applied to distinguish PD and SWEDD based on group-wise differences. Results. Four connections showed significant group-wise differences and correlated with the Unified Parkinson's Disease Rating Scale sponsored by the Movement Disorder Society. The SVM classifier attained 77.92\% accuracy in distinguishing between SWEDD and PD using these identified connections. Conclusions. The connections and regions identified represent candidates for future research investigations.
\end{abstract}

\section{Introduction}

Parkinson's disease (PD), the second most common neurodegenerative disease in the elderly, causes the loss of dopaminergic neurons in the brainstem, especially those in the substantia nigra [1]. Characteristic PD symptoms include resting tremor, rigidity, impaired voluntary movement, and cognitive impairment [2]. PD is typically diagnosed by criteria such as those of the United Kingdom's Parkinson's Disease Society Brain Bank [3]. However, about 10\% of clinically diagnosed PD patients do not lose dopaminergic neurons in the brainstem and are classified as scans without evidence of dopaminergic deficit (SWEDD) [4]. SWEDD is a nuclear medicine term referring to patients with PD that show no evidence of dopamine transporter deficit. The etiology of SWEDD has been under debate; some consider it an early phase of PD, while others consider it a different disease [5]. Distinguishing between PD and SWEDD is important, as the two groups are potentially different and thus may require different therapeutic strategies.

Diffusion tensor imaging (DTI), an MRI imaging technique, quantifies in vivo neuronal fiber information using anisotropic water diffusion in the white matter. This approach has been used to differentiate between normal controls (NCs) and PD patients [6-8]. DTI has a few limitations and is unable to distinguish between efferent and afferent connections; however, it is the only practical option for assessing in vivo fiber information [9]. Raw data from DTI need to be processed with an algorithm in a process known as tractography so that relevant fiber information can be extracted [10]. Extracted fiber information is further analyzed with connectivity analysis, which is suitable for analyzing complex objects such as the brain. Connectivity derived from DTI is known as structural connectivity, because DTI is related to actual neuronal fiber connections between regions. Many studies have also derived brain connectivity 
information from resting state functional MRI (fMRI), which measures local brain activity based on the blood oxygen leveldependent (BOLD) effect [11].

Connectivity analysis requires that regions of interest (ROIs) are specified in order to explore correlations or connections among the regions. These ROIs can be specified by coregistering information from a predefined parcellation of the brain, which can be derived from structural or functional information [12]. Brain connectivity has been explored for analysis of motor-related regions previously known to be affected by PD [13]. Structural connectivity of corticocortical, cortical-subcortical, and subcortical connections in cortico-basal ganglia-thalamocortical (CBTC) connections was investigated in this study. Connectivity measures can be used to quantify group-wise differences and as biomarkers of important clinical variables in PD research. Many studies have successfully established group-wise differences between PD patients and NCs based on neuroimaging [2, 6-8, 13-15]. However, less attention has been paid to differences between PD and SWEDD patients. Thus, the focus of this study was to quantify differences between PD and SWEDD patients based on connectivity information from DTI tractography.

We obtained diffusion MRI data from the Parkinson's disease Progressive Marker Institute (PPMI) research database [16]. Connectivity analysis was applied to PD and SWEDD patients. Group-wise differences between PD and SWEDD patients were quantified using the number of neuronal fibers connecting regions as features. We tried to identify connections between regions with significant group-wise differences in terms of the number of neuronal fibers and then find significant correlations between the number of fibers that connected specific regions and the Movement Disorder Society-sponsored Unified Parkinson's Disease Rating Scale (MDS-UPDRS).

\section{Material and Methods}

2.1. Subjects. Our study was a retrospective analysis of anonymized imaging data, and local IRB approval was obtained. The PPMI is an observational clinical study to evaluate PD and other closely related cohorts using advanced imaging, biological sampling, and clinical and behavioral assessments to identify potential biomarkers of progression of PD [16]. The PPMI includes a database where imaging and clini$\mathrm{cal} /$ behavioral assessments data are shared with the scientific community. The study included 423 de novo PD, 64 SWEDD, and 196 normal control cases as of July 2015. Our study explored subjects with MRI imaging data aside from the usual SPECT. Our SWEDD group was limited to 37 SWEDD patients who underwent DTI and structural MRI imaging. We included a total of 77 participants and classified them into SWEDD $(n=37)$ and $\operatorname{PD}(n=40)$ groups that were matched according to age and sex ratio. Subgroups were classified based on PPMI consortium guidelines [16]. Detailed criteria for the SWEDD group are as follows. First, patients had to demonstrate at least two of the following symptoms: resting tremor, bradykinesia, rigidity, or either asymmetric resting tremor or bradykinesia. Second, patients had to have been diagnosed with PD for 2 years or less at the
TABLE 1: Participant information: values are reported as mean \pm standard deviation (SD).

\begin{tabular}{lccc}
\hline & SWEDD & PD & $P$ value \\
\hline Number of subjects & $n=37$ & $n=40$ & - \\
Age (years) & $61.3 \pm 10.9$ & $59.5 \pm 11.0$ & 0.403 \\
Sex (male/female) & $24 / 13$ & $21 / 19$ & 0.264 \\
Disease duration (months) & $6.4 \pm 7.9$ & $5.58 \pm 4.7$ & 0.587 \\
Hoehn \& Yahr score & $1.6 \pm 0.5$ & $1.5 \pm 0.5$ & 0.921 \\
MDS-UPDRS-III score & $14.0 \pm 9.1$ & $18.6 \pm 8.1$ & 0.752 \\
GDS score & $3.3 \pm 3.7$ & $2.6 \pm 3.1$ & 0.392 \\
MoCA score & $27.4 \pm 1.8$ & $28.1 \pm 2.1$ & 0.103 \\
\hline
\end{tabular}

time of screening, with no evidence of dopamine transporter deficit on dopamine transporter SPECT imaging. Third, patients had to have been free of PD medication for at least 6 months. Fourth, patients were 30 years or older at the time of PD diagnosis. Participant information, including scores of MDS-UPDRS, Montreal Cognitive Assessment (MoCA), and Geriatric Depression Scale (GDS), is given in Table 1. The disease duration of all $(n=422)$ available PD patients in the PPMI database was $6.57 \pm 6.51$ months (mean \pm SD), which was not significantly different from the PD group used in our study $(P=0.343)$. The imaging data of PD and SWEDD were from baseline scans of patients not using any PD-related medication.

2.2. Imaging Data. We obtained diffusion and T1- and T2-weighted MRI data from the PPMI database [16]. We obtained T1- and T2-weighted MR imaging in addition to the DTI data because the image preprocessing steps were required in T1- and T2-weighted MR imaging. T1- and T2-weighted MRI data were obtained using the following parameters: $\mathrm{TR}=2300 \mathrm{~ms}, \mathrm{TE}=2.98 \mathrm{~ms}, \mathrm{TI}=900 \mathrm{~ms}$, image matrix $=240 \times 256 \times 176$, and voxel resolution $=1 \times 1$ $\times 1 \mathrm{~mm}^{3}$. Parameters for the T2-weighted images were as follows: $\mathrm{TR}=3000 \mathrm{~ms}, \mathrm{TE}=101 \mathrm{~ms}$, image matrix $=228 \times$ $256 \times 48$, and voxel resolution $=0.9375 \times 0.9375 \times 3 \mathrm{~mm}^{3}$. Diffusion images were obtained with a Siemens $3 \mathrm{~T}$ scanner using the following parameters: 3T scanner, $b=1000 \mathrm{~s} / \mathrm{mm}^{2}$, 64 diffusion gradient directions with one $b 0$ image, image matrix $=116 \times 116 \times 72$, and voxel resolution $=1.98 \times 1.98$ $\times 2 \mathrm{~mm}^{3}$.

2.3. Image Preprocessing. Preprocessing steps were performed to extract fiber information from DTI data. Readers interested in details of the preprocessing steps are referred to a review article [17]. Briefly, image preprocessing was performed using the Connectome Mapping Toolkit (CMTK), a Python-based open-source software (http://www.cmtk.org/). T1- and T2-weighted images were aligned to the nondiffusion-weighted image $(b 0)$ by a nonlinear registration using FSL for each subject [18]. The registered T1-weighted image was segmented into white matter, grey matter, and cerebrospinal fluid using FreeSurfer [19]. The segmented white matter was then used to guide the tractography algorithm. An overview of image preprocessing procedures is provided in Figure 1. 
TABLE 2: Motor-related regions that were explored for connectivity.

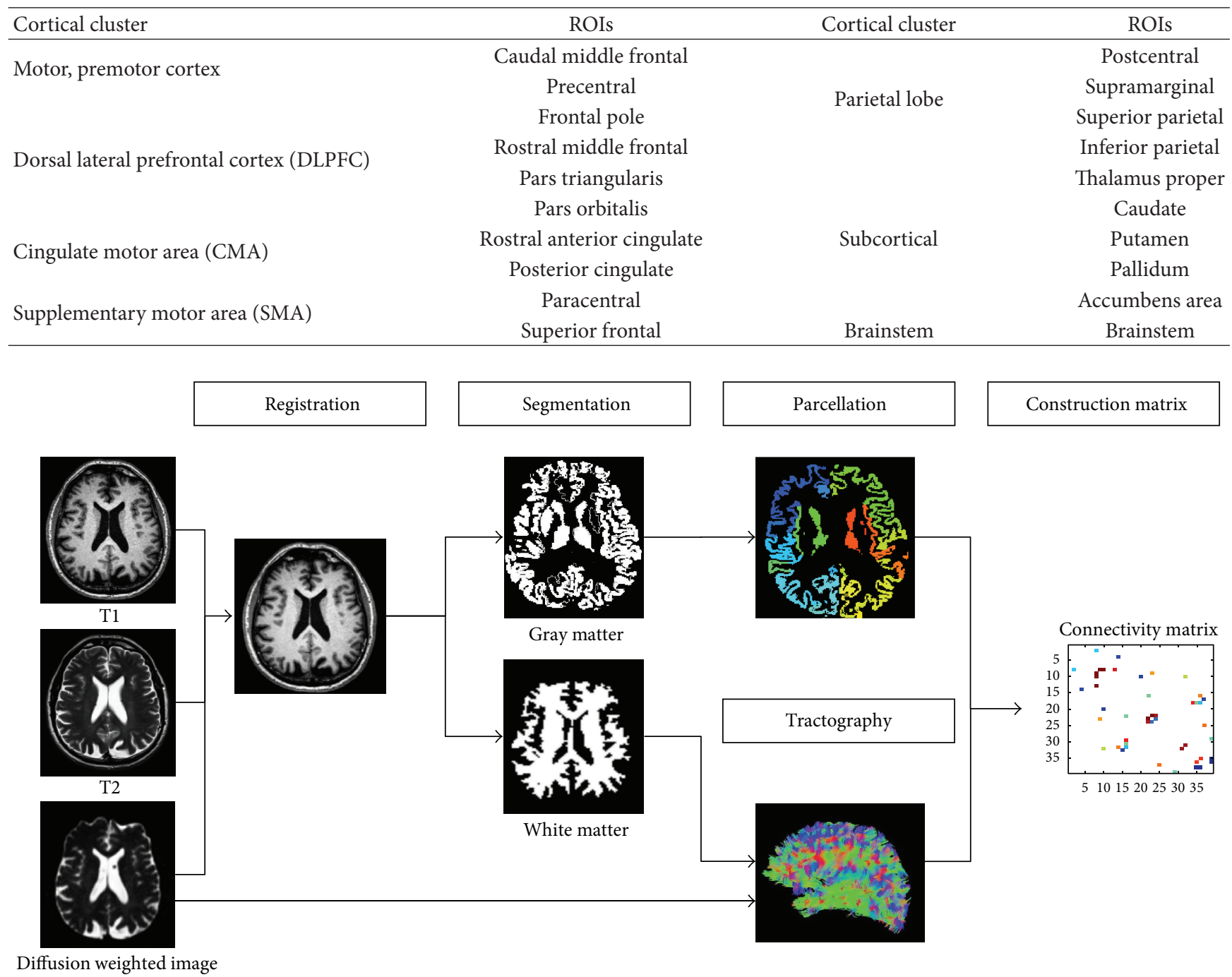

FIGURE 1: Overall image preprocessing procedures.

2.4. ROI Specifications. Exploring connectivity requires that the ROIs are specified so that correlations among them can be investigated. We coregistered and transferred information from a predefined atlas onto the individual subject image spaces to specify the ROIs using the DesikanKilliany anatomical atlas [12]. Our analysis focused on the 39 motor-related regions known to be affected by PD. A full list of explored regions is provided in Table 2 [13]. The regions include the motor cortex, premotor cortex, dorsal lateral prefrontal cortex (DLPFC), cingulate motor area, supplementary motor area (SMA), and a few regions in the parietal lobe, subcortex, and brainstem. The corticocortical connections mediate hand, face, foot, and trunk motor activity [20]. The CBTC connection has been shown to be important in $\mathrm{PD}$ patients [21]. Fiber connections from the brainstem were included to explore the initiation and control of movement [22]. Connections from the brainstem were considered because this structure is densely connected with the pallidum and substantia nigra, which are important regions affected by $\mathrm{PD}$. Connections among these regions were explored to quantify structural connectivity of corticocortical, cortical-subcortical, and subcortical connections.

2.5. Tractography and Network Construction. Eddy currentinduced distortions and head motion were corrected with FSL [18]. Fiber information was computed using the FACT algorithm implemented within the Diffusion Toolkit and CMTK software [17]. The FACT algorithm propagated a line from the center of a seed voxel along the direction of the dominant vector, which was determined by the largest eigenvector of the tensor. The starting point of the next voxel was the intercept of the previous voxel. Tracking terminated when the algorithm reached a region with an abrupt change in fiber direction (i.e., angle threshold greater than $60^{\circ}$ ). Tracking was limited to white matter and its neighbors, as neuronal fibers mainly exist in the white matter. Every voxel was considered a seed voxel, and we retained only fibers whose end points were within the predefined ROIs. 


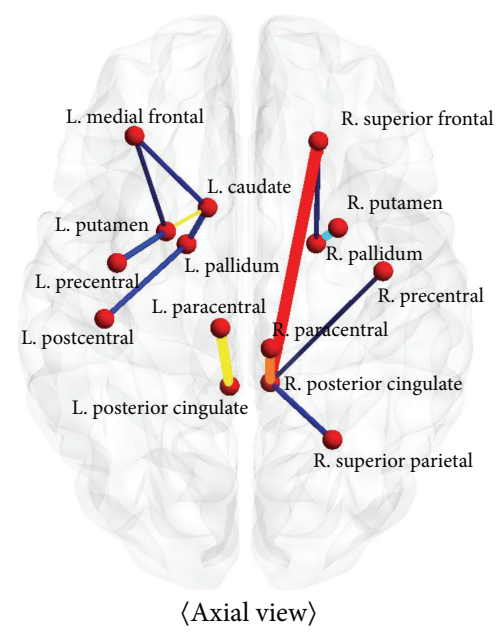

(a)

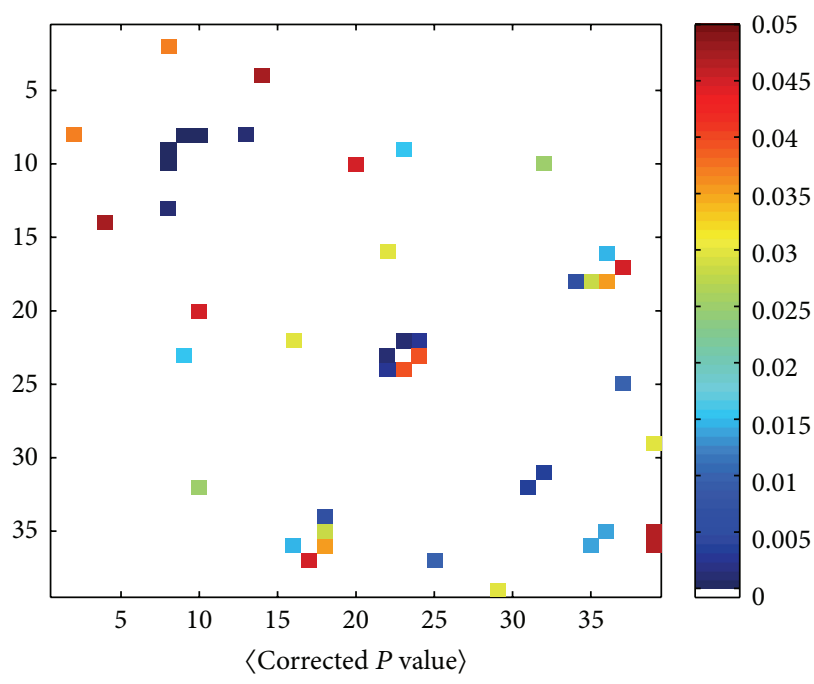

(b)

FIGURE 2: Connections and group-wise differences. Significantly different connections were rendered (a), and corrected $P$ values were plotted (b).

Connectivity was assessed using nodes and edges of a graph. Nodes were assigned ROIs that were transferred from the predefined parcellation. Each edge value was assumed to be the number of fibers whose end points resided within two ROIs, which was entered into the matrix as an element. Elements with less than 5 fibers were excluded, as identification of a very small number of fibers was considered to be an unstable result of the tractography algorithm [23]. The matrix was referred to as the connectivity matrix. We adopted a simple network model that considered undirected and weighted edges.

2.6. Statistical Tests. Group-wise differences between SWEDD and PD groups were explored in 39 regions. For each group, the connectivity matrices of the two groups (i.e., PD and SWEDD) were stacked into two three-dimensional matrices. Each element in the stacked connectivity matrix contained many observations (i.e., 37 or 40 observations), which were tested using nonparametric permutation tests to distinguish between PD and SWEDD. Permutation tests were performed by randomly assigning PD and SWEDD subjects 10,000 times. One permutation involved randomly assigning the first 37 cases to the SWEDD group and the remaining 40 cases to the PD group. Differences in the number of fibers were deemed significant if they did not belong to the 95th percentile of the null distribution derived from the permutation tests $(P<0.05$, corrected $)$.

2.7. Correlation with Clinical Scores. We performed correlation analysis to detect possible links between structural connectivity and clinical scores. We pooled connectivity matrices between the groups (i.e., PD and SWEDD) into one long vector and then computed Spearman correlations with MDS-UPDRS scores for all identified connections.
The Holm-Bonferroni correction was adopted to compute corrected $P$ values in order to reveal significantly correlated connections.

2.8. Classification Using Identified Connections. The four significant connections were fed into a support vector machine (SVM) classifier framework with linear kernels to separate between SWEDD and PD. The technical details of the SVM are found in a review by Vapnik [24]. We applied the leaveone-out-cross validation method for discrimination of training and test data because of the limited number of available subjects. For example, given 37 SWEDD and 40 PD cases, we assigned one case as the test case and used the remaining 76 cases as the training data for the SVM classifier. The process was repeated 77 times, choosing a different test case each time. The SVM classifier seeks a decision boundary that can effectively separate samples near the decision boundary. Classifier accuracy, sensitivity, and specificity were computed by comparing the classifier outcomes with known ground truth using MATLAB [25].

\section{Results}

3.1. Structural Connectivity Differences. The stacked connectivity matrices of SWEDD and PD groups were investigated using nonparametric permutation tests in order to identify elements (i.e., connections between regions) that distinguished between PD and SWEDD patients. Twentythree connections were significantly different between PD and SWEDD, as shown in Figure 2 and Table 3. Of these connections, 11 increased in PD compared to SWEDD and 12 decreased in PD compared to SWEDD. Overall, eight connections were significant, with corrected $P$ values less than 0.01 . Of these connections, three had $P$ values less than 0.001 , 
TABLE 3: Identified connections with significant group-wise differences. Significantly different connections are reported in terms of connectivity values and corrected $P$ values. Connectivity values are reported as mean \pm standard deviation (SD). Bold text shows identified connections with significant differences (corrected $P<0.001$ ) between PD and SWEDD.

\begin{tabular}{|c|c|c|c|c|}
\hline & \multicolumn{4}{|c|}{ Group-wise differences } \\
\hline & \multirow{2}{*}{ Connection } & \multicolumn{2}{|c|}{ Number of fibers (mean \pm SD) } & \multirow{2}{*}{ Corrected $P$ value } \\
\hline & & $\mathrm{PD}$ & SWEDD & \\
\hline \multirow{11}{*}{ (a) Cortico-cortical connection } & L_precentral-L_posteriorcingulate & $4.6 \pm 8.23$ & $1.56 \pm 2.89$ & 0.028 \\
\hline & L_paracentral-L_superiorfrontal & $1.18 \pm 1.67$ & $3.21 \pm 6.29$ & 0.039 \\
\hline & L_paracentral-L_posteriorcingulate & $8.72 \pm 8.32$ & $3.48 \pm 4.35$ & $<0.001$ \\
\hline & L_posteriorcingulate-L_superiorfrontal & $11.7 \pm 8.96$ & $6.27 \pm 6.23$ & 0.003 \\
\hline & L_parsorbitalis-R_superiorfrontal & $0.65 \pm 3.33$ & $2.18 \pm 4.72$ & 0.046 \\
\hline & R_paracentral-L_paracentral & $0.65 \pm 2.02$ & $2.59 \pm 4.5$ & 0.014 \\
\hline & R_paracentral-R_posteriorcingulate & $37.7 \pm 23.47$ & $17.8 \pm 23.3$ & $<0.001$ \\
\hline & R_precentral-R_posteriorcingulate & $13.1 \pm 16.75$ & $5.54 \pm 14.3$ & 0.034 \\
\hline & R_posteriorcingulate-R_superiorfrontal & $43.67 \pm 27.54$ & $19.94 \pm 21.7$ & $<0.001$ \\
\hline & R_posteriorcingulate-R_superiorparietal & $10.45 \pm 17.27$ & $3.00 \pm 6.78$ & 0.005 \\
\hline & R_rostralmiddlefrontal-R_inferiorparietal & $1.42 \pm 3.20$ & $0.29 \pm 1.17$ & 0.045 \\
\hline \multirow{7}{*}{ (b) Cortico-subcortical connection } & L_pallidum-L_frontalpole & $0.77 \pm 1.51$ & $2.83 \pm 6.40$ & 0.045 \\
\hline & L_pallidum-L_postcentral & $5.52 \pm 9.17$ & $16.08 \pm 26.3$ & 0.012 \\
\hline & L_putamen-L_precentral & $14.5 \pm 8.95$ & $24.86 \pm 24.48$ & 0.013 \\
\hline & L_putamen-L_rostralmiddlefrontal & $34.87 \pm 30.86$ & $21.83 \pm 21.34$ & 0.035 \\
\hline & L_caudate-L_rostralmiddlefrontal & $37.5 \pm 41.4$ & $15.59 \pm 24.66$ & 0.027 \\
\hline & L_thalamusproper-L_rostralmiddlefrontal & $7.27 \pm 9.75$ & $3.27 \pm 5.02$ & 0.007 \\
\hline & R_pallidum-R_superiorfrontal & $1.50 \pm 2.76$ & $4.57 \pm 7.97$ & 0.022 \\
\hline \multirow{5}{*}{ (c) Subcortical connection } & L_caudate-L_putamen & $2.28 \pm 3.34$ & $5.59 \pm 7.58$ & 0.009 \\
\hline & R_putamen-R_pallidum & $2.28 \pm 2.33$ & $4.51 \pm 3.98$ & 0.002 \\
\hline & Brainstem-L_putamen & $3.80 \pm 5.71$ & $7.78 \pm 10.96$ & 0.046 \\
\hline & Brainstem-L_caudate & $2.00 \pm 4.29$ & $4.51 \pm 6.46$ & 0.048 \\
\hline & Brainstem-R_thalamusproper & $31.48 \pm 18.72$ & $42.08 \pm 23.13$ & 0.029 \\
\hline
\end{tabular}

including the connections between the paracentral gyrus and posterior cingulate gyrus in the left hemisphere, between the posterior cingulate gyrus and superior frontal lobe in the right hemisphere, and between the posterior cingulate gyrus and paracentral gyrus in the right hemisphere. Further details regarding identified connections are reported in Table 3.

\subsection{Correlations between Identified Connections and Clinical} Scores. Correlation analysis was performed to identify possible links between structural connectivity and MDS-UPDRSIII score. The approach was similar to that in the previous section, which sought connections that distinguished two groups, except that, in this case, we sought connections that were significantly correlated with MDS-UPDRS score. Spearman's correlation was adopted because clinical scores are categorical measurements. Figure 3 shows significantly correlated connections with MDS-UPDRS score in the 39 ROIs. Only a few of the identified connections with groupwise differences were observed to be significantly correlated (corrected $P<0.001$ ) with MDS-UPDRS score, as shown in bold text in Table 4. Four connections had significant groupwise differences in structural connectivity and correlation with clinical scores. Clinical scores were correlated with the connection between the precentral gyrus and putamen in the left hemisphere $(r=0.54$, corrected $P<0.001)$, between the posterior cingulate gyrus and superior frontal lobe in the right hemisphere $(r=0.54$, corrected $P<0.001)$, between the posterior cingulate gyrus and paracentral gyrus in the right hemisphere $(r=0.56$, corrected $P<0.001)$, and between the paracentral gyrus and superior frontal lobe in the left hemisphere $(r=-0.32$, corrected $P<0.001)$.

3.3. Classifier Performance. The SVM classifier using linear kernels was applied to separate SWEDD and PD cases. Accuracy, sensitivity, and specificity of the classifier are reported in Table 5 for distinguishing between PD and SWEDD cases. Overall, the classification results were good (sensitivity, specificity, and accuracy were $78.38 \%, 77.50 \%$, and $77.92 \%$, resp.).

\section{Discussion}

We showed that PD and SWEDD patients might be distinguished using connectivity values derived from fiber connections. The four connections that distinguished between PD 


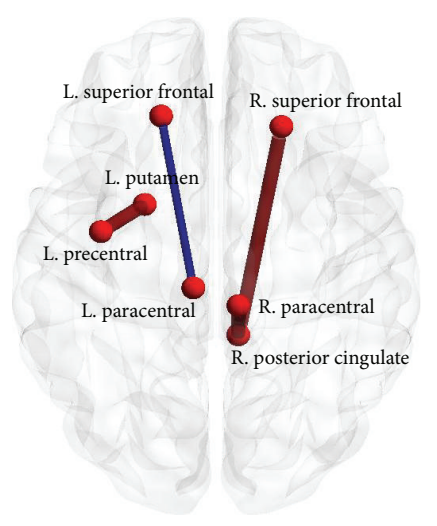

$\langle$ Axial view $\rangle$

(a)

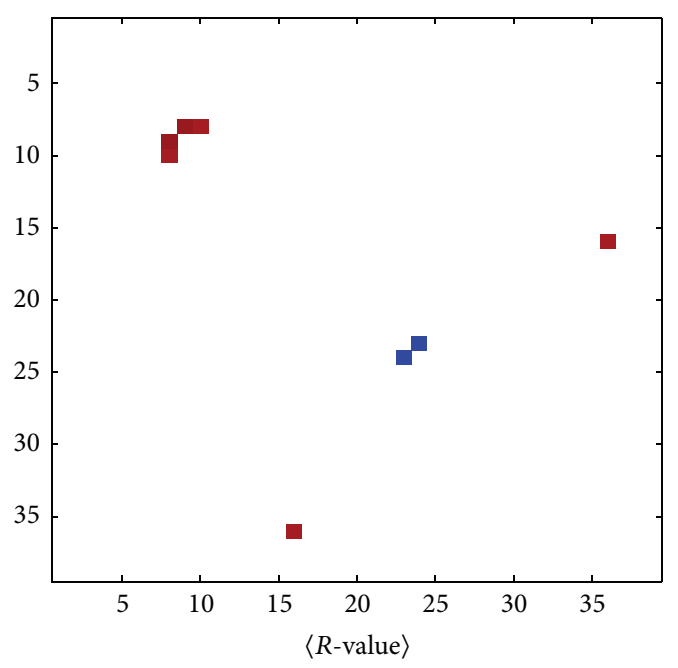

(b)

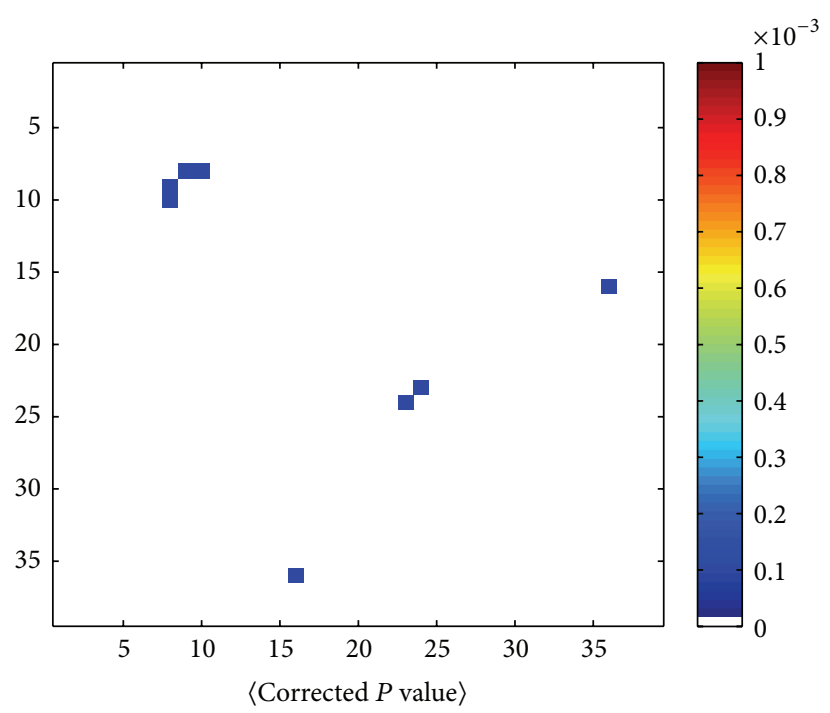

(c)

FIGURE 3: Connections and correlations with MDS-UPDR-III. The significantly correlated connections were rendered (a). $R$-values (b) and corrected $P$ values (c) of significantly correlated connections with MDS-UPDRS-III (corrected $P<0.001$ ) are plotted.

and SWEDD also correlated well with clinical scores, indicating their relevance. Furthermore, our study successfully classified between PD and SWEDD using these identified connections. This was a proof of concept study, however, and our findings should be interpreted cautiously in terms of clinical application. Further research with more samples is needed to validate our findings.

Among cortico-cortical connections, bilateral paracentral-posterior cingulate, precentral-posterior cingulate, and posterior cingulate-superior frontal connections showed decreased connectivity in SWEDD compared to PD patients. These results are consistent with a recent MRI study $[13,26$, 27]. The connections and related regions that were previously reported to have increased functional connectivity for PD patients are in the sensorimotor cortex, SMA, cingulate gyrus, primary motor cortex, and parietal cortex [13]. Similar connections and regions were identified using diffusionweighted MRI in PD patients [27]. A previous study reported reduced fractional anisotropy (FA) values in the frontal lobe, premotor area, and cingulate in PD patients [26]. Among subcortical-cortical connections, seven showed significant group-wise differences. Pallidum-frontal pole, pallidumpostcentral gyrus, and putamen-precentral gyrus connections in the left hemisphere decreased in PD compared to SWEDD. Rostral middle frontal gyrus-putamen, rostral middle frontal gyrus-caudate, and rostral middle frontal gyrus-thalamus connections increased in PD compared to SWEDD. These results corroborate previous studies using fMRI and diffusion MRI in PD patients $[8,13]$. A decrease in functional connectivity using fMRI was reported in the left putamen, SMA, and DLPFC in PD patients compared to NCs [13]. Other studies using diffusion MRI have shown that FA values in the putamen and caudate are lower in PD compared to NCs [8]. Within subcortical connections, five were significantly different between PD and SWEDD, in agreement with existing studies $[8,28-30]$. Structural connectivity studies of PD patients have shown a reduction in connectivity in the nigrostriatal tract (connections 
TABLE 4: Correlation with clinical scores was investigated. Structural connectivity values based on the number of fibers connecting two regions are reported within the identified connections. Correlations between structural connectivity and MDS-UPDSR score are reported as Spearman correlation coefficients, with corrected $P$ values in the rightmost column. Bold text shows the connections that significantly correlated with MDS-UPDRS-III (corrected $P<0.001$ ).

Correlation with MDS-UPDRS-III score

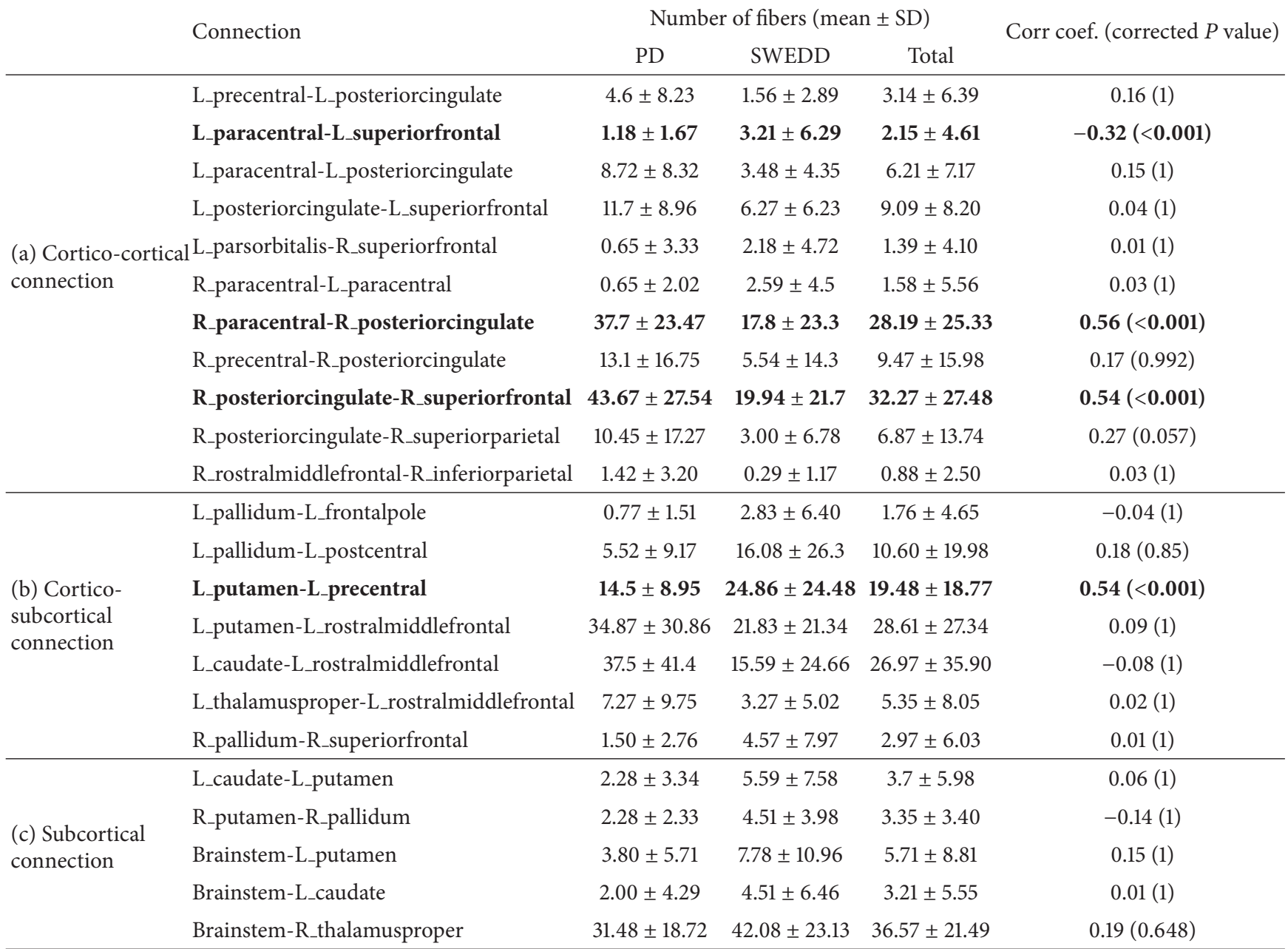

TABLE 5: Classifier performance to distinguish between PD and SWEDD.

\begin{tabular}{lccc}
\hline Group & Sensitivity (\%) & Specificity (\%) & Accuracy (\%) \\
\hline SWEDD versus PD & 78.38 & 77.50 & 77.92 \\
\hline
\end{tabular}

among SN, STN, putamen, and pallidum) using DTI [28]. Others report decreased FA values in the substantia nigra, caudate, and putamen in PD patients [8]. One functional connectivity study showed a decreased connection in the pallidum-putamen using resting state functional MRI (rsfMRI) [29]. PD patients also showed decreased fiber counts in SN-putamen and pallidum connections [30].

Existing connectivity studies have mainly explored differences between NCs and PD patients [8, 13, 26-31]. There is a lack of comparable studies comparing SWEDD and PD using structural connectivity. The previous discussion contrasting our findings with existing literature was largely derived from comparisons of PD patients and NCs, not necessarily PD and SWEDD. The existing literature describes various MRI techniques, including resting state fMRI, structural MRI, and diffusion MRI [8, 13, 26-31]. Comparisons between our findings and existing research should consider the imaging modalities used in those studies. Some researchers have adopted non-MRI and non-SPECT approaches to studying PD and SWEDD [32, 33]. One study successfully adopted electrophysiological tremor parameters to distinguish between tremor-dominant PD and SWEDD [32]. In addition, a sonography study attained $85 \%$ accuracy in distinguishing PD from SWEDD [33].

Our results showed significant connections between subcortical and cortical structures mostly in left intrahemisphere connections, as shown in Table 3. One possible explanation might be related to lateralized dopamine transporter 
TABLE 6: Correlations between DaT imaging measures and MDS-UPDRS score.

\begin{tabular}{lcccc}
\hline & Left caudate SBR & Left putamen SBR & Right caudate SBR & Right putamen SBR \\
\hline$R$-value & -0.33 & -0.33 & -0.29 & -0.36 \\
$P$ value & 0.003 & 0.003 & 0.011 & 0.001 \\
\hline
\end{tabular}

uptake in PD. Previous studies using $\mathrm{I}^{123}$-IBZM SPECT revealed lateralized difference in the striatal uptake of $\mathrm{I}^{123}$ IBZM, which implies lateralized availability of dopamineD2 receptors [34]. Another possible explanation might be the handedness of patients. Our study considered largely right-handed patients, which could be one factor leading to the lateralized connectivity patterns shown in this study. Some studies have found that right-handed PD patients have significantly lower dopamine transporter uptake in the left putamen compared to the right putamen [31]. Thus, lateralized connectivity differences in our study might be related to right-handed PD patients with reduced dopamine uptake in the left striatum. Side of disease onset in PD patients might differentially affect connectivity patterns $[35,36]$. A previous study reported that an association between rightsided symptoms in $\mathrm{PD}$ and depression was related to the side of disease onset [37]. Unfortunately, the PPMI database lacked side of disease onset information; thus, we could not quantify the difference between sides of disease onset.

DaT imaging by definition can distinguish between PD and SWEDD $[4,16]$. Our study showed that an MRIbased measure (i.e., DTI tractography) could also distinguish between PD and SWEDD. MRI has advantages over SPECT, as it is more readily available at clinical sites and does not expose patients to radiation. Our results using DTI showed better correlation with clinical variables such as MDS-UPDRS III. We reported a correlation value of 0.54 between DTI results and MDS-UPDRS, while correlation between DaT imaging measures (i.e., striatal binding ratio (SBR) of the caudate and putamen) and MDS-UPDRS from the same PPMI data showed correlation values ranging from -0.36 to -0.29 , as shown in Table 6 .

SWEDD patients are heterogeneous, with many different underlying conditions. Modern MRI techniques can quantify diffusion (as shown in DTI), perfusion, and other tissue properties [38-40]. Applying various MRI techniques to SWEDD patients could reveal differences that relate to various underlying conditions of SWEDD such as essential tremor and dystonic tremor. Unfortunately, the PPMI database simply did not have enough samples in the SWEDD group to subdivide them into different subgroups based on underlying condition. We leave this important topic for future research, which might be feasible as the PPMI collects more cases.

Our study has some limitations. First, DTI itself suffers from limitations including (1) inability to distinguish between efferent and afferent connections and (2) capacity to account for only major fiber tracts due to limited voxel resolution. Adopting an advanced form of DTI known as high-angular resolution diffusion imaging (HARDI) would allow for complex modeling within a voxel; however, HARDI requires longer scan times compared to DTI [41]. Second, multimodal imaging could be adopted to better explore the brain network. For example, we could have used additional fMRI to complement the DTI data [42]. Multimodal analysis of the brain network would allow incorporation of complementary information derived from different modalities in order to better quantify differences between PD and SWEDD. Third, our study only considered SWEDD and PD, leaving out normal controls. Future studies need to consider all three groups (i.e., PD, SWEDD, and NCs groups) so that the identity of SWEDD can be better established.

\section{Conclusion}

We identified four structural connections that distinguished between PD and SWEDD and also correlated well with MDS-UPDRS score. These findings confirm correlations between important connections and well-established clinical scores. Our study successfully distinguished between PD and SWEDD using these identified connections. Thus, our findings might serve as a first to step to investigate whether SWEDD patients have a unique connectivity profile.

\section{Conflict of Interests}

The authors declare no conflict of interests regarding this study.

\section{Acknowledgments}

This study was supported in part by the Institute for Basic Science (grant no. IBS-R015-D1, http://www.ibs.re.kr/). Imaging data were obtained from the PPMI, which is a public-private partnership funded by the Michael J. Fox Foundation for Parkinson's Research and funding partners, including Abbott, Avid Pharmaceuticals, Biogen Idec, Bristol-Myers Squibb, Covance, Elan, GE Healthcare, Genentech, GlaxoSmithKline, Lilly, Merck, Meso Scale Discovery, Pfizer, Roche, and UCB (http://www.ppmi-info.org/fundingpartners).

\section{References}

[1] J. A. Obeso, M. C. Rodríguez-Oroz, B. Benitez-Temino et al., "Functional organization of the basal ganglia: therapeutic implications for Parkinson's disease," Movement Disorders, vol. 23, supplement 3, pp. S548-S559, 2008.

[2] A. J. Stoessl, "Neuroimaging in Parkinson's disease," Neurotherapeutics, vol. 8, no. 1, pp. 72-81, 2011.

[3] I. Litvan, K. P. Bhatia, D. J. Burn et al., "SIC task force appraisal of clinical diagnostic criteria for parkinsonian disorders," Movement Disorders, vol. 18, no. 5, pp. 467-486, 2003.

[4] S. A. Schneider, M. J. Edwards, P. Mir et al., "Patients with adultonset dystonic tremor resembling Parkinsonian tremor have scans without evidence of dopaminergic deficit (SWEDDs)," Movement Disorders, vol. 22, no. 15, pp. 2210-2215, 2007. 
[5] A. Batla, R. Erro, M. Stamelou et al., "Patients with scans without evidence of dopaminergic deficit: a long-term follow-up study," Movement Disorders, vol. 29, no. 14, pp. 1820-1825, 2014.

[6] G. Gattellaro, L. Minati, M. Grisoli et al., "White matter involvement in idiopathic Parkinson disease: a diffusion tensor imaging study," American Journal of Neuroradiology, vol. 30, no. 6, pp. 1222-1226, 2009.

[7] K. Zhang, C. Yu, Y. Zhang et al., "Voxel-based analysis of diffusion tensor indices in the brain in patients with Parkinson's disease," European Journal of Radiology, vol. 77, no. 2, pp. 269273, 2011.

[8] K. Yoshikawa, Y. Nakata, K. Yamada, and M. Nakagawa, "Early pathological changes in the parkinsonian brain demonstrated by diffusion tensor MRI," Journal of Neurology, Neurosurgery \& Psychiatry, vol. 75, no. 3, pp. 481-484, 2004.

[9] P. Hagmann, J.-P. Thiran, L. Jonasson et al., "DTI mapping of human brain connectivity: statistical fibre tracking and virtual dissection," NeuroImage, vol. 19, no. 3, pp. 545-554, 2003.

[10] M. Descoteaux, R. Deriche, T. R. Knösche, and A. Anwander, "Deterministic and probabilistic tractography based on complex fibre orientation distributions," IEEE Transactions on Medical Imaging, vol. 28, no. 2, pp. 269-286, 2009.

[11] S. M. Smith, "The future of FMRI connectivity," NeuroImage, vol. 62 , no. 2, pp. 1257-1266, 2012.

[12] R. S. Desikan, F. Ségonne, B. Fischl et al., "An automated labeling system for subdividing the human cerebral cortex on MRI scans into gyral based regions of interest," NeuroImage, vol. 31, no. 3, pp. 968-980, 2006.

[13] T. Wu, L. Wang, Y. Chen, C. Zhao, K. Li, and P. Chan, "Changes of functional connectivity of the motor network in the resting state in Parkinson's disease," Neuroscience Letters, vol. 460, no. 1, pp. 6-10, 2009.

[14] C. M. Ellis, G. Lemmens, S. C. R. Williams et al., "Changes in putamen $\mathrm{N}$-acetylaspartate and choline ratios in untreated and levodopa-treated Parkinson's disease: a proton magnetic resonance spectroscopy study," Neurology, vol. 49, no. 2, pp. 438-444, 1997.

[15] G. Tedeschi, I. Litvan, S. Bonavita et al., "Proton magnetic resonance spectroscopic imaging in progressive supranuclear palsy, Parkinson's disease and corticobasal degeneration," Brain, vol. 120, no. 9, pp. 1541-1552, 1997.

[16] K. Marek, D. Jennings, S. Lasch et al., “The Parkinson Progression Marker Initiative (PPMI)," Progress in Neurobiology, vol. 95, no. 4, pp. 629-635, 2011.

[17] A. Daducci, S. Gerhard, A. Griffa et al., “The connectome mapper: an open-source processing pipeline to map connectomes with MRI," PLoS ONE, vol. 7, no. 12, Article ID e48121, 2012.

[18] S. M. Smith, M. Jenkinson, M. W. Woolrich et al., "Advances in functional and structural MR image analysis and implementation as FSL," NeuroImage, vol. 23, supplement 1, pp. S208-S219, 2004.

[19] B. Fischl, "FreeSurfer," NeuroImage, vol. 62, no. 2, pp. 774-781, 2012.

[20] J. D. Schmahmann and D. Pandya, Fiber Pathways of the Brain, OUP, New York, NY, USA, 2009.

[21] A. O. Ceballos-Baumann, "Functional imaging in Parkinson's disease: activation studies with PET, fMRI and SPECT," Journal of Neurology, Supplement, vol. 250, supplement 1, pp. I15-I23, 2003.

[22] D. Nandi, T. Z. Aziz, X. Liu, and J. F. Stein, "Brainstem motor loops in the control of movement," Movement Disorders, vol. 17, supplement 3, pp. S22-S27, 2002.
[23] P. Fillard, M. Descoteaux, A. Goh et al., "Quantitative evaluation of 10 tractography algorithms on a realistic diffusion MR phantom," NeuroImage, vol. 56, no. 1, pp. 220-234, 2011.

[24] V. N. Vapnik, "An overview of statistical learning theory," IEEE Transactions on Neural Networks, vol. 10, no. 5, pp. 988-999, 1999.

[25] Guide MUs, MathWorks, Natick, Mass, USA, 1998.

[26] A. T. Karagulle Kendi, S. Lehericy, M. Luciana, K. Ugurbil, and P. Tuite, "Altered diffusion in the frontal lobe in Parkinson disease," American Journal of Neuroradiology, vol. 29, no. 3, pp. 501-505, 2008.

[27] H. J. Kim, S. J. Kim, H. S. Kim et al., "Alterations of mean diffusivity in brain white matter and deep gray matter in Parkinson's disease," Neuroscience Letters, vol. 550, pp. 64-68, 2013.

[28] Y. Zhang, I.-W. Wu, S. Buckley et al., "Diffusion tensor imaging of the nigrostriatal fibers in Parkinson's disease," Movement Disorders, vol. 30, no. 9, pp. 1229-1236, 2015.

[29] M. Sharman, R. Valabregue, V. Perlbarg et al., "Parkinson's disease patients show reduced cortical-subcortical sensorimotor connectivity," Movement Disorders, vol. 28, no. 4, pp. 447-454, 2013.

[30] W.-Q. Tan, C.-S. Yeoh, H. Rumpel et al., "Deterministic tractography of the nigrostriatal-nigropallidal pathway in Parkinson's disease," Scientific Reports, vol. 5, article 17283, 2015.

[31] C. Scherfler, K. Seppi, K. J. Mair et al., "Left hemispheric predominance of nigrostriatal dysfunction in Parkinson's disease," Brain, vol. 135, no. 11, pp. 3348-3354, 2012.

[32] P. Schwingenschuh, D. Ruge, M. J. Edwards et al., "Distinguishing SWEDDs patients with asymmetric resting tremor from Parkinson's disease: a clinical and electrophysiological study," Movement Disorders, vol. 25, no. 5, pp. 560-569, 2010.

[33] H. Stockner, P. Schwingenschuh, A. Djamshidian et al., "Is transcranial sonography useful to distinguish scans without evidence of dopaminergic deficit patients from Parkinson's disease?" Movement Disorders, vol. 27, no. 9, pp. 1182-1185, 2012.

[34] M. B. Knable, D. W. Jones, R. Coppola et al., "Lateralized differences in iodine-123-IBZM uptake in the basal ganglia in asymmetric Parkinson's disease," Journal of Nuclear Medicine, vol. 36, no. 7, pp. 1216-1225, 1995.

[35] C. D. Good, I. Johnsrude, J. Ashburner, R. N. A. Henson, K. J. Friston, and R. S. J. Frackowiak, "Cerebral asymmetry and the effects of sex and handedness on brain structure: a voxel-based morphometric analysis of 465 normal adult human brains," NeuroImage, vol. 14, no. 3, pp. 685-700, 2001.

[36] K. C. Stewart, H. H. Fernandez, M. S. Okun, R. L. Rodriguez, C. E. Jacobson, and C. J. Hass, "Side onset influences motor impairments in Parkinson disease," Parkinsonism \& Related Disorders, vol. 15, no. 10, pp. 781-783, 2009.

[37] D. Weintraub, A. B. Newberg, M. S. Cary et al., "Striatal dopamine transporter imaging correlates with anxiety and depression symptoms in Parkinson's disease," Journal of Nuclear Medicine, vol. 46, no. 2, pp. 227-232, 2005.

[38] P. Hagmann, O. Sporns, N. Madan et al., "White matter maturation reshapes structural connectivity in the late developing human brain," Proceedings of the National Academy of Sciences of the United States of America, vol. 107, no. 44, pp. 19067-19072, 2010.

[39] P. J. Basser, S. Pajevic, C. Pierpaoli, J. Duda, and A. Aldroubi, "In vivo fiber tractography using DT-MRI data," Magnetic Resonance in Medicine, vol. 44, no. 4, pp. 625-632, 2000. 
[40] E. T. Petersen, T. Lim, and X. Golay, "Model-free arterial spin labeling quantification approach for perfusion MRI," Magnetic Resonance in Medicine, vol. 55, no. 2, pp. 219-232, 2006.

[41] A. W. Anderson, "Measurement of fiber orientation distributions using high angular resolution diffusion imaging," Magnetic Resonance in Medicine, vol. 54, no. 5, pp. 1194-1206, 2005.

[42] P. Staempfli, C. Reischauer, T. Jaermann, A. Valavanis, S. Kollias, and P. Boesiger, "Combining fMRI and DTI: a framework for exploring the limits of fMRI-guided DTI fiber tracking and for verifying DTI-based fiber tractography results," NeuroImage, vol. 39, no. 1, pp. 119-126, 2008. 


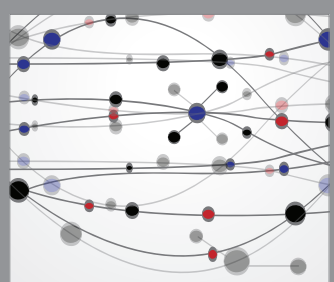

The Scientific World Journal
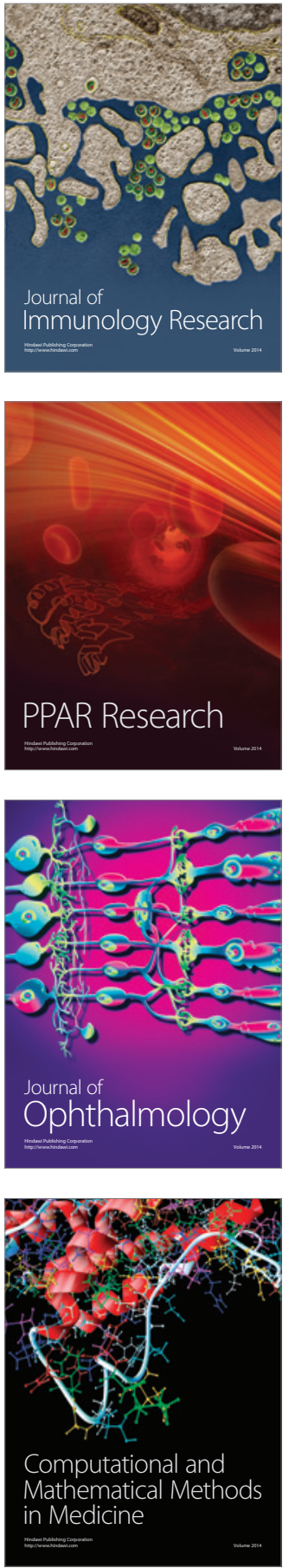

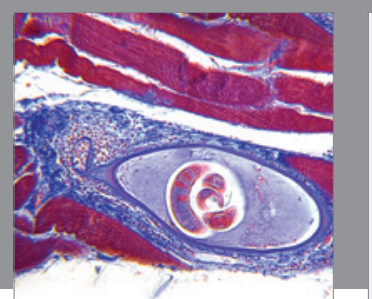

Gastroenterology Research and Practice

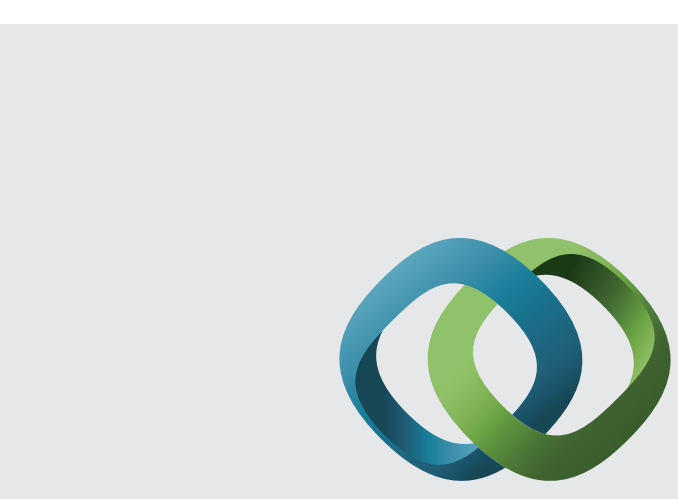

\section{Hindawi}

Submit your manuscripts at

http://www.hindawi.com
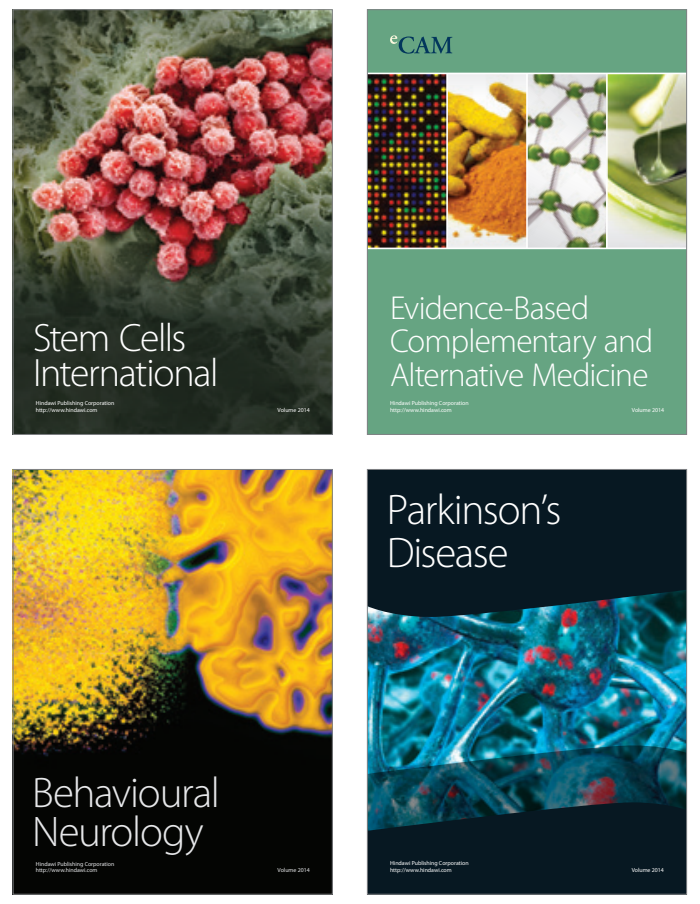
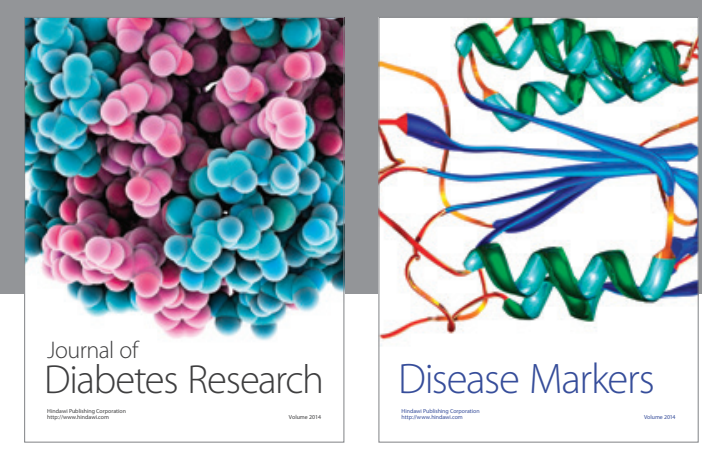

Disease Markers
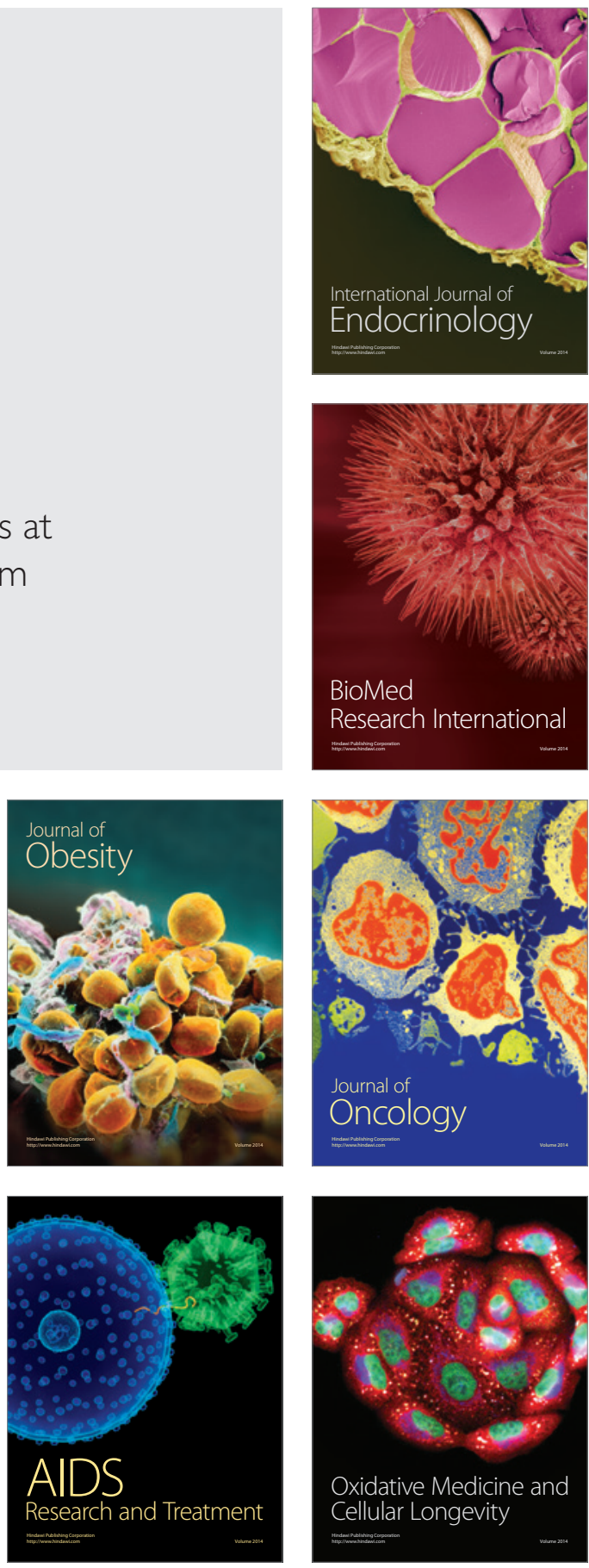